\title{
Essay on Fractional Riemann-Liouville Integral Operator versus Mikusinski's
}

\author{
Ming $\mathrm{Li}^{1}$ and Wei Zhao ${ }^{2}$ \\ ${ }^{1}$ School of Information Science \& Technology, East China Normal University, No. 500 Dongchuan Road, Shanghai 200241, China \\ ${ }^{2}$ Department of Computer and Information Science, University of Macau, Avenue Padre Tomas Pereira, Taipa, Macau \\ Correspondence should be addressed to Ming Li; ming_lihk@yahoo.com
}

Received 23 April 2013; Accepted 7 May 2013

Academic Editor: Ezzat G. Bakhoum

Copyright (c) 2013 M. Li and W. Zhao. This is an open access article distributed under the Creative Commons Attribution License, which permits unrestricted use, distribution, and reproduction in any medium, provided the original work is properly cited.

\begin{abstract}
This paper presents the representation of the fractional Riemann-Liouville integral by using the Mikusinski operators. The Mikusinski operators discussed in the paper may yet provide a new view to describe and study the fractional Riemann-Liouville integral operator. The present result may be useful for applying the Mikusinski operational calculus to the study of fractional calculus in mathematics and to the theory of filters of fractional order in engineering.
\end{abstract}

\section{Introduction}

Fractional calculus gains increase interests in processing biomedical signals; see, for example, [1-16]. The fractional integral of the Riemann-Liouville type is widely used in the field; see, for example, [17-22].

Denote by $C(0, \infty)$ the set of piecewise continuous functions on $(0, \infty)$. Let $v>0$ and $f(t) \in C(0, \infty)$. Assume that $f(t)$ is integrable on any finite subinterval of $[0, \infty)$. For $t>0$, denote by ${ }_{0} D_{t}^{-v}$ the fractional RiemannLiouville integral operator of order $v$ [19]. Then, the fractional Riemann-Liouville integral of order $v$ of $f(t)$ is given by

$$
{ }_{0} D_{t}^{-v} f(t)=\frac{1}{\Gamma(v)} \int_{0}^{t}(t-u)^{v-1} f(u) d u,
$$

where $\Gamma(\cdot)$ is the gamma function. As early as 1919 , O'Shaughnessy and Post studied the problem indexed by 433 [23]. The desired solution to Problem 433 is the solution to the differential equation of order $1 / 2$ expressed by

$$
\frac{d^{1 / 2} f(t)}{d t^{1 / 2}}-\frac{f(t)}{t}=0
$$

The above needs the differential of order $1 / 2$. They gave the following solution to (2) based on the fractional RiemannLiouville integral [24]:

$$
f(t)=C t^{-1 / 2} \exp \left(-\frac{1}{t}\right),
$$

where $C$ is a constant.

This short paper aims at exhibiting that ${ }_{0} D_{t}^{-v}$ is equivalent to the Mikusinski operator $l^{\nu}$. The significance of our analysis is as follows. Since the algebra properties of the Mikusinski operators are satisfactorily studied and well known, see, for example, [25-28], one may immediately infer that the algebra properties of ${ }_{0} D_{t}^{-v}$ are consistent with the Mikusinski operators. Moreover, the present result suggests that the Mikusinski operators may be used for studying differential equations or filters of fractional order in signal processing.

The remainder of this paper is organized as follows. We will derive (1) from the point of view of the Mikusinski operators in Section 2. Discussions are given in Section 3, which is followed by conclusions.

\section{Derivation}

In this section, we will first brief the Mikusinski operators. Then, the derivation of (1) is given based on the Mikusinski operators. 
The Mikusinski operators are described by convolution [28-30]. Let $a(t)$ and $b(t)$ belong to $C(0, \infty)$. Following the usage of Mikusinski's, we rewrite $a(t)$ and $b(t)$ by

$$
a=\{a(t)\}, \quad b=\{b(t)\} .
$$

The convolution described by Mikusinski is then given by

$$
a b=\{a(t)\}\{b(t)\}=\left\{\int_{0}^{t} a(t-\tau) b(\tau) d \tau\right\} .
$$

The deconvolution, therefore, is expressed by

$$
\frac{a}{b}=\frac{\{a(t)\}}{\{b(t)\}} .
$$

Define $l=\{1\}$ such that

$$
\{1\}\{a(t)\}=\left\{\int_{0}^{t} a(\tau) d \tau\right\} .
$$

The representations (5) and (6) may be convenient to study the operations of the convolution and its inverse from a view of algebra. For instance, that $C(0, \infty)$ is a commutative ring is obvious.

Let $a=\{1\}$ in (7). Then,

$$
l^{2}=\{1\}\{1\}=\left\{\int_{0}^{t} d \tau\right\}=\left\{\frac{t}{1}\right\} .
$$

In the general case of $n=1, \ldots$, one has

$$
l^{n}=\left\{\frac{t^{n-1}}{(n-1) !}\right\}
$$

where $0 !=1$. The above $l^{n}$ may be termed as a Mikusinski operator.

When one exerts $l^{n}$ on $f(t) \in C(0, \infty)$, that is, $l^{n}\{f(t)\}$, the following Cauchy formula results:

$$
l^{n}\{f(t)\}=\left\{\int_{0}^{t} \frac{(t-\tau)^{n-1}}{(n-1) !} f(\tau) d \tau\right\} .
$$

Considering the generalization of $l^{n}$ in (9) for $v>0$ yields another Mikusinski operator given by

$$
l^{v}=\left\{\frac{t^{v-1}}{(v-1) !}\right\}=\left\{\frac{t^{\nu-1}}{\Gamma(v)}\right\} .
$$

Further, by taking into account $l^{v}\{f(t)\}$, we have

$$
l^{v}\{f(t)\}=\left\{\int_{0}^{t} \frac{(t-\tau)^{v-1}}{\Gamma(v)} f(\tau) d \tau\right\} .
$$

Releasing the usage of Mikusinski in $\{\cdot\}$ for the purpose of his operational calculus, we have

$$
l^{v} f(t)=\int_{0}^{t} \frac{(t-\tau)^{v-1}}{\Gamma(v)} f(\tau) d \tau
$$

This completes the derivation because (13) is the definition of the fractional Riemann-Liouville integral of order $v$.

\section{Discussions}

From (1) and (13), one sees that the fractional RiemannLiouville integral operator of order $v$, that is, ${ }_{0} D_{t}^{-v}$, is equivalent to the Mikusinski operator $l^{v}$ though the originality of Mikusinski's by introducing $l^{v}$ may be for the purpose of his theory of operational calculus.

On the one hand, we recall that the Mikusinski operational calculus is a useful tool for studying differential equations. On the other hand, $l^{v}$ may yet be an alternative of ${ }_{0} D_{t}^{-v}$, so that the Mikusinski operational calculus may be expanded into the field of differential equations and signal processing of fractional order, which attracts interest in biomedical engineering; see, for example, [31].

We note that $f(t) \in C(0, \infty)$ is not necessary in (1). In fact, (1) exists if $f(t)$ is a generalized function [32]. In addition, $f(t)$ may be a random function such as the Brownian motion [33]. Due to the consistence of ${ }_{0} D_{t}^{-v}$ with $l^{v}$, one sees that a generalized function $f(t)$ in (13) may also be allowed. Finally, we should remember that the fractional Riemann-Liouville integral operator may be extended to its corresponding, more precisely, the fractional Riemann-Liouville differential operator if $v<0$. This may correspond to the deconvolution in the Mikusinsiki's operational calculus, which we will work on in the future. Finally, we mention that possible applications of the Mikusinsiki's operational calculus to other issues, for example, in [34-36], may be interesting.

\section{Conclusions}

We have exhibited that the fractional Riemann-Liouville integral operator may be expressed by using the Mikusinsiki operators, giving another outlook of the fractional RiemannLiouville integral operator. Thus, we have noticed that the Mikusinski operational calculus may yet be a tool for studying differential equations or systems of fractional order.

\section{Acknowledgments}

This work was supported in part by the 973 plan under the project Grant no. 2011CB302800 and by the National Natural Science Foundation of China under the project Grant nos. 61272402,61070214 , and 60873264.

\section{References}

[1] F. Liu and K. Burrage, "Novel techniques in parameter estimation for fractional dynamical models arising from biological systems," Computers and Mathematics with Applications, vol. 62, no. 3, pp. 822-833, 2011.

[2] R. L. Magin, "Fractional calculus models of complex dynamics in biological tissues," Computers and Mathematics with Applications, vol. 59, no. 5, pp. 1586-1593, 2010.

[3] W. G. Glöckle and T. F. Nonnenmacher, "A fractional calculus approach to self-similar protein dynamics," Biophysical Journal, vol. 68, no. 1, pp. 46-53, 1995.

[4] B. J. West, "Fractal physiology and the fractional calculus: a perspective," Frontiers in Fractal Physiology, vol. 1, article 12, 2010. 
[5] E. G. Bakhoum and C. Toma, "Specific mathematical aspects of dynamics generated by coherence functions," Mathematical Problems in Engineering, vol. 2011, Article ID 436198, 10 pages, 2011.

[6] C. A. Monje, Y. Q. Chen, B. M. Vinagre, D. Xue, and V. Feliu, Fractional Order Systems and Controls-Fundamentals and Applications, Springer, 2010.

[7] H. Sun, Y. Chen, and W. Chen, "Random-order fractional differential equation models," Signal Processing, vol. 91, no. 3, pp. 525-530, 2011.

[8] C. Toma, "Advanced signal processing and command synthesis for memory-limited complex systems," Mathematical Problems in Engineering, vol. 2012, Article ID 927821, 13 pages, 2012.

[9] S. V. Muniandy, W. X. Chew, and C. S. Wong, "Fractional dynamics in the light scattering intensity fluctuation in dusty plasma," Physics of Plasmas, vol. 18, no. 1, Article ID 013701, 2011.

[10] J. Yang, Z. Chen, W. S. Chen, and Y. Chen, "Robust affine invariant descriptors," Mathematical Problems in Engineering, vol. 2011, Article ID 185303, 15 pages, 2011.

[11] H. M. Srivastava and Ž. Tomovski, "Fractional calculus with an integral operator containing a generalized Mittag-Leffler function in the kernel," Applied Mathematics and Computation, vol. 211, no. 1, pp. 198-210, 2009.

[12] C. Cattani, G. Pierro, and G. Altieri, "Entropy and multifractality for the myeloma multiple TET 2 gene," Mathematical Problems in Engineering, vol. 2012, Article ID 193761, 14 pages, 2012.

[13] I. S. Jesus and J. A. Tenreiro Machado, "Application of integer and fractional models in electrochemical systems," Mathematical Problems in Engineering, vol. 2012, Article ID 248175, 17 pages, 2012.

[14] J. A. Tenreiro MacHado, M. F. Silva, R. S. Barbosa et al., "Some applications of fractional calculus in engineering," Mathematical Problems in Engineering, vol. 2010, Article ID 639801, 34 pages, 2010.

[15] C. Cattani, M. Scalia, E. Laserra, I. Bochicchio, and K. K. Nandi, "Correct light deflection in Weyl conformal gravity," Physical Review D, vol. 87, no. 4, Article ID 47503, 4 pages, 2013.

[16] C. H. Eab and S. C. Lim, "Fractional Langevin equations of distributed order," Physical Review E, vol. 83, no. 3, Article ID 031136, 2011.

[17] C. Cattani, "Fractional calculus and shannon wavelet," Mathematical Problems in Engineering, vol. 2012, Article ID 502812, 26 pages, 2012.

[18] S. Castellucci and M. Carlini, "Modelling and simulation for energy production parametric dependence in greenhouses," Mathematical Problems in Engineering, vol. 2010, Article ID 590943, 28 pages, 2010.

[19] K. S. Miller and B. Ross, An Introduction to the Fractional Calculus and Fractional Differential Equations, John Wiley \& Sons, 1993.

[20] S. V. Muniandy and S. C. Lim, "Modeling of locally self-similar processes using multifractional Brownian motion of RiemannLiouville type," Physical Review E, vol. 63, no. 4, pp. 461041461047, 2001.

[21] S. C. Lim and S. V. Muniandy, "On some possible generalizations of fractional Brownian motion," Physics Letters A, vol. 266, no. 2-3, pp. 140-145, 2000.

[22] J. Klafter, S. C. Lim, and R. Metzler, Fractional Dynamics: Recent Advances, World Scientific, 2012.
[23] L. O'Shaughnessy and E. L. Post, "Problem 433," The American Mathematical Monthly, vol. 25, no. 4, pp. 172-173, 1918.

[24] L. O'Shaughnessy and E. L. Post, "Discussion of problem 433," The American Mathematical Monthly, vol. 26, no. 1, pp. 37-39, 1919.

[25] R. A. Struble, "Analytical and algebraic aspects of the operational calculus," SIAM Review, vol. 19, no. 3, pp. 403-436, 1977.

[26] R. G. Buschman, "The algebraic derivative of Mikusinski," The American Mathematical Monthly, vol. 74, no. 6, pp. 717-718, 1967.

[27] D. A. Klarner, "Algebraic theory for difference and differential equations," The American Mathematical Monthly, vol. 76, no. 4, pp. 366-373, 1969.

[28] J. Mikusinski, Operational Calculus, Pergamon Press, 1959.

[29] T. K. Boehme, “The Convolution integral," SIAM Review, vol. 10, no. 4, pp. 407-416, 1968.

[30] G. Bengochea and L. Verde-Star, "Linear algebraic foundations of the operational calculi," Advances in Applied Mathematics, vol. 47, no. 2, pp. 330-351, 2011.

[31] H. Sheng, Y. Q. Chen, and T. S. Qiu, Fractional Processes and Fractional Order Signal Processing, Springer, 2012.

[32] I. M. Gelfand and K. Vilenkin, Generalized Functions, vol. 1, Academic Press, New York, NY, USA, 1964.

[33] B. B. Mandelbrot and J. W. van Ness, "Fractional Brownian motions, fractional noises and applications," SIAM Review, vol. 10, no. 4, pp. 422-437, 1968.

[34] M. Carlini, T. Honorati, and S. Castellucci, "Photovoltaic greenhouses: comparison of optical and thermal behaviour for energy savings," Mathematical Problems in Engineering, vol. 2012, Article ID 743764, 10 pages, 2012.

[35] E. G. Bakhoum and C. Toma, "Mathematical transform of traveling-wave equations and phase aspects of quantum interaction," Mathematical Problems in Engineering, vol. 2010, Article ID 695208, 15 pages, 2010.

[36] J. W. Yang, Y. J. Chen, and M. Scalia, "Construction of affine invariant functions in spatial domain," Mathematical Problems in Engineering, vol. 2012, Article ID 690262, 11 pages, 2012. 


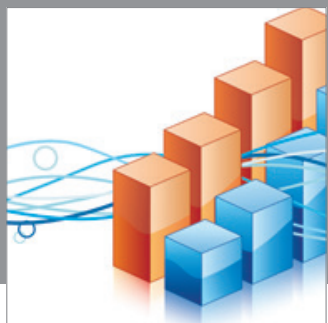

Advances in

Operations Research

mansans

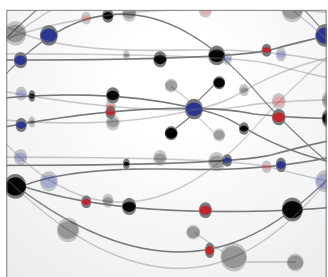

The Scientific World Journal
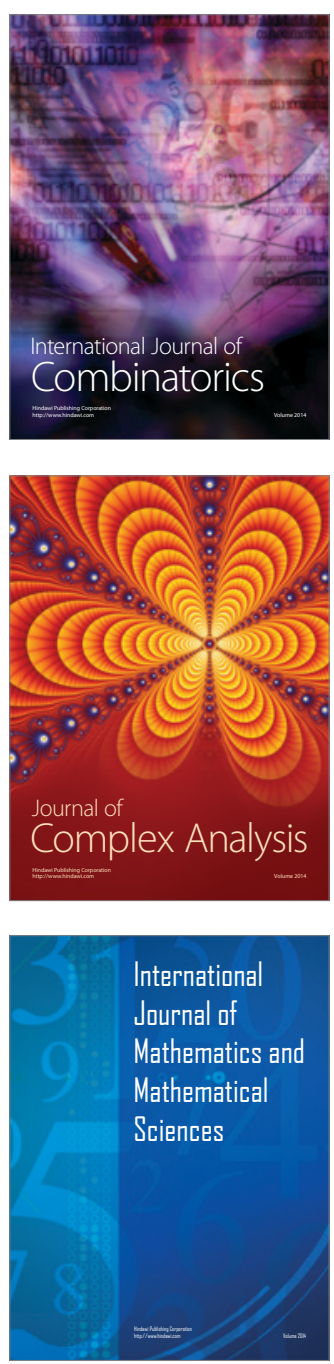
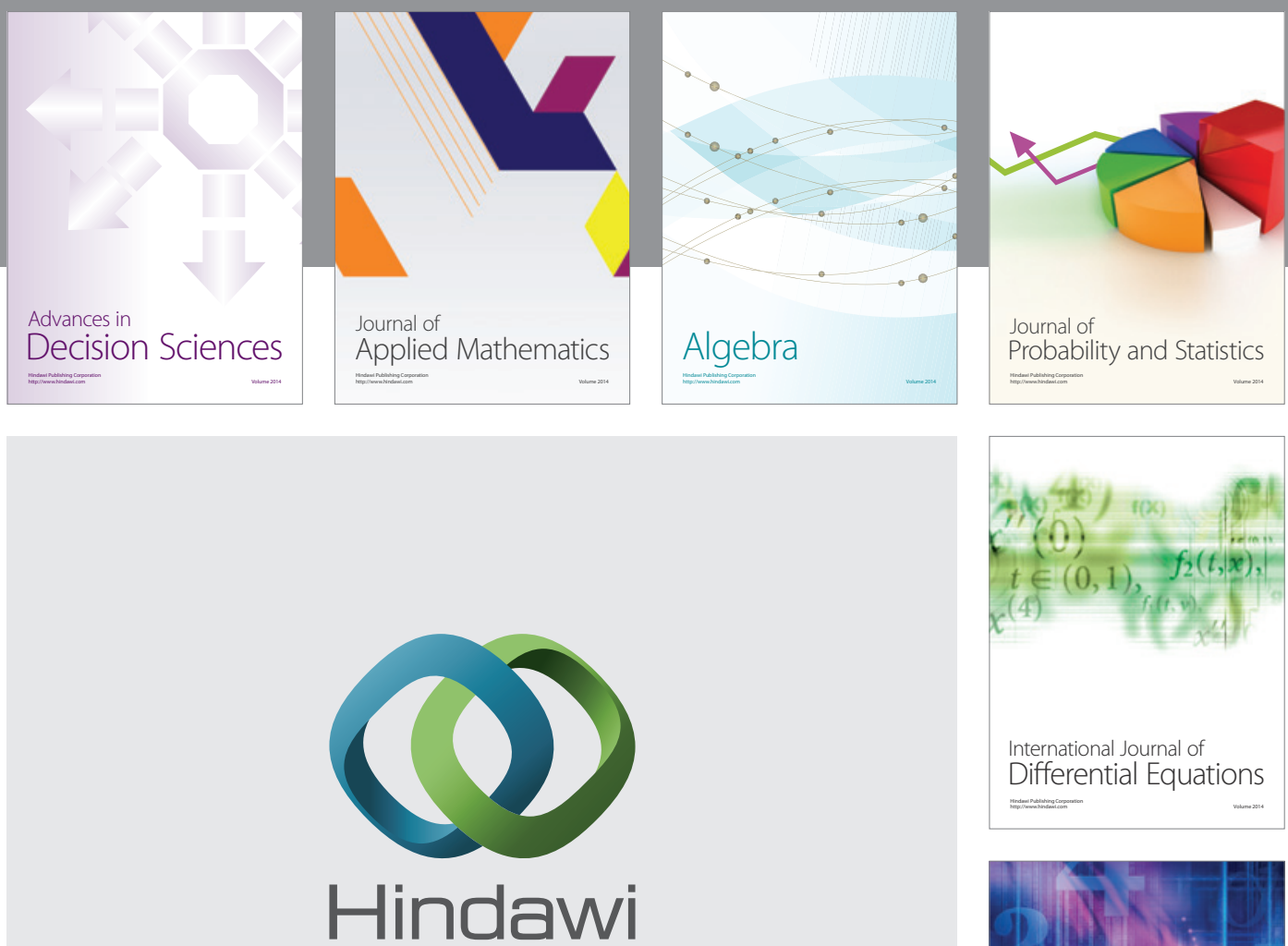

Submit your manuscripts at http://www.hindawi.com
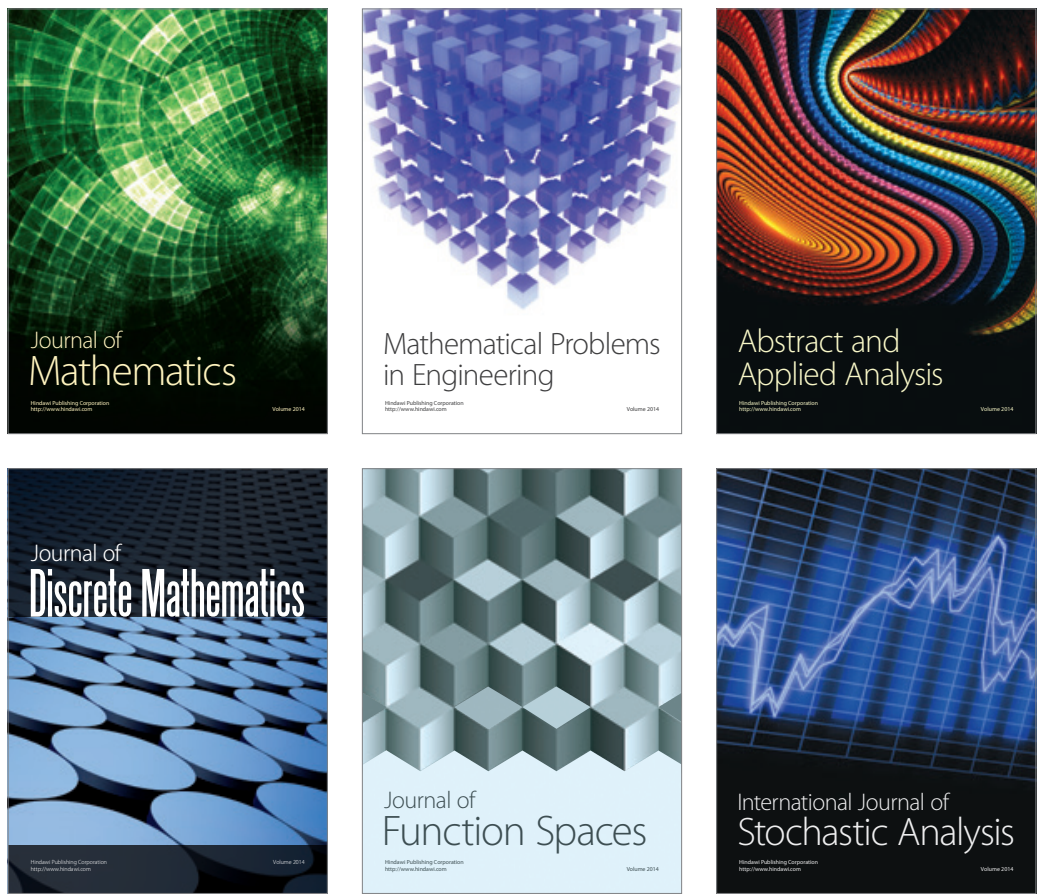

Journal of

Function Spaces

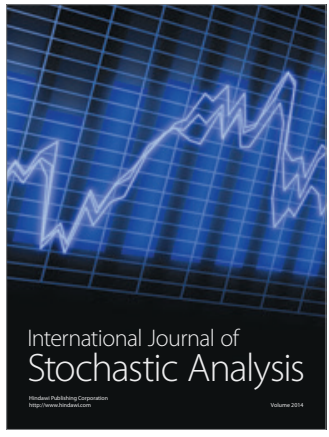

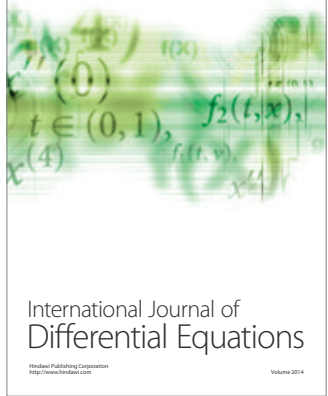
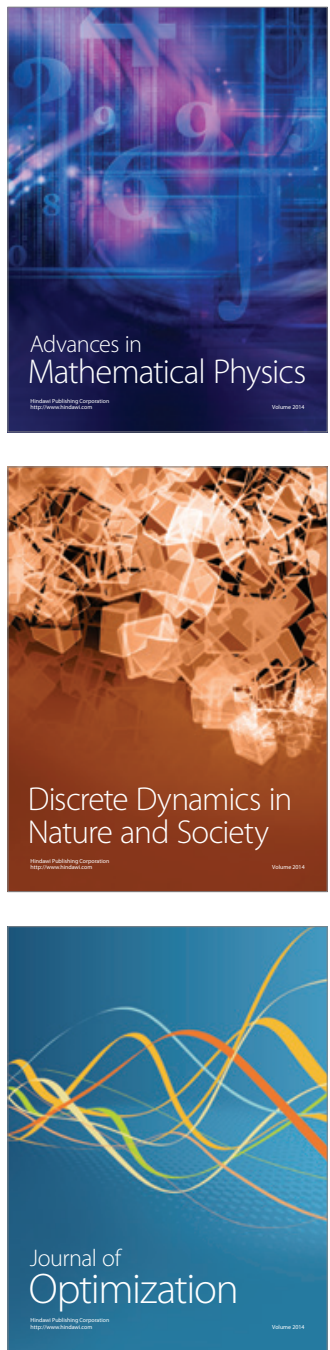